Check for updates

Cite this: RSC Adv., 2017, 7, 43300

Received 3rd August 2017

Accepted 24th August 2017

DOI: $10.1039 / \mathrm{c} 7 \mathrm{ra0} 8600 \mathrm{~h}$

rsc.li/rsc-advances

\section{Electrospun API-loaded mixed matrix membranes for controlled release}

\author{
B. Castro-Dominguez, ${ }^{a}$ K. Moroney, ${ }^{\text {ab }}$ B. Schaller, ${ }^{a}$ S. O'Connor, ${ }^{a}$ A. Cloonan, ${ }^{\text {ac }}$
} T. T. N. Vo, ${ }^{b}$ G. Walker ${ }^{a}$ and E. J. O'Reilly (D) *a

The development of biocompatible membrane materials capable of delivering active pharmaceutical ingredients (APIs) over a fixed time period offers significant advantages to the pharmaceutical and biomedical industries alike. In addition the incorporation of APIs within polymeric materials potentially allows for the formation of amorphous solid dispersions (ASDs), which have shown enhanced bioavailability, increased dissolution profiles and enhanced adsorption into the blood stream. Mixed matrix membranes (MMMs) have been at the forefront of such developments, however manufacturing MMMs with consistent batch to batch physical characteristics has proved challenging thereby significantly impeding the use of such materials by the pharmaceutical sector. This article describes the development, for the first time, of API and molecular sieve loaded mixed matrix membranes (MMMs) via electrospinning techniques. The developed membranes displayed consistent and controllable physical properties and more efficient API release relative to membranes prepared using traditional casting techniques. Mathematical modelling disclosed that the membranes generated via electrospinning show excellent correlation between experimental and predicted API release kinetics thereby paving the way for the development of MMMs for both pharmaceutical and biomedical applications.

\section{Introduction}

Mixed Matrix Membranes (MMMs) have primarily been investigated for gas and liquid separation ${ }^{\mathbf{1 , 2}}$ however active pharmaceutical ingredient (API) loaded membranes have recently been reported as advanced controlled drug delivery devices., ${ }^{\mathbf{3} 4}$ Table 1 shows various API-based mixed matrix membrane (MMM) systems that have demonstrated faster and more efficient delivery of the API than conventional polymeric membranes. MMMs are typically polymeric films that contain dispersed fillers, often inorganic in nature, such as zeolites and metal organic frameworks (MOFs) that aim to not only control the API delivery/separatory properties of the membrane but also increase mechanical strength. ${ }^{5}$ The encapsulation of molecules in zeolites has been previously performed such as the case of $\alpha$-tocopheryl acetate into zeolite Y for textile applications. ${ }^{6}$

MMMs are typically synthesized utilizing a casting methodology whereby the precursor solution is poured onto a plate followed by solvent removal via evaporation. Membrane production via casting has many drawbacks including batch to batch inconsistencies and has previously been described as

${ }^{a}$ Synthesis and Solid State Pharmaceutical Centre (SSPC), Bernal Institute, University of Limerick, Limerick, Ireland. E-mail: Emmet.OReilly@ul.ie; Tel: +35361234663 ${ }^{b}$ MACSI, Department of Mathematics and Statistics, University of Limerick, Limerick, Ireland

${ }^{c}$ Irish Composites Centre, Bernal Institute, University of Limerick, Limerick, Ireland "more of an art than a science". ${ }^{7}$ This is highly undesirable for pharmaceutical or biomedical applications whereby delivery of a predefined concentration of API or precise control of membrane diameter may be required. As an alternative to casting, electrospinning permits the controlled fabrication of fibres, which can be subsequently arranged into membranes. ${ }^{\mathbf{8} 9}$ Electrospinning uses electrostatic forces to produce micrometer sized fibres from polymer solutions or melts and has been widely used for applications including tissue engineering, ${ }^{\mathbf{1 0}}$ biomedical sensing ${ }^{11}$ and the formation of composite materials ${ }^{\mathbf{1 2 - 1 5}}$ as outlined in Table 2. The incorporation of zeolites and metal organic frameworks (MOFs) into polymeric materials via electrospinning has previously been demonstrated by Cheng et $a l .{ }^{13}$ who prepared nonwoven polyimide/silica hybrid nanofiberous fabrics $(<100 \mathrm{~nm}$ in diameter) for filtration applications. In addition Ostermann et al. successfully incorporated the MOF ZIF-8 into PVP fibres for gas sensing applications. ${ }^{\mathbf{1 4}}$

Table 1 Mixed matrix membrane/API systems reported in the literature

\begin{tabular}{lll}
\hline Type of membrane & API tested & Reference \\
\hline PDMS-NaX & Gemfibrozil & 15 \\
PDMS-NaX & Ibuprofen & 3 \\
PDMS-NaX & Tramadol & 16 \\
PVDF-NaY & Ibuprofen & 20
\end{tabular}


Table 2 ; Mixed matrix membranes via electrospinning

\begin{tabular}{|c|c|c|c|}
\hline Poly(vinyl alcohol)/silica & None & Fabric & 12 \\
\hline Polyimide/silica & None & Fabric & 13 \\
\hline Polyurethane/CNTs & None & Fabric & 14 \\
\hline Polyvinylpyrrolidone/ZIF-8 & None & Fibres & 15 \\
\hline Poly(E-caprolactone)/silica & Tetracycline hydrochloride & Scaffold for tissue & 10 \\
\hline
\end{tabular}

Despite advances in gas sensing and filtration applications, the use of electrospinning as a fabrication technique for MMMs for pharmaceutical and biomedical applications remains to be exploited as can be seen in Table 2 .

The aim of this work is twofold, firstly, to exploit electrospinning as a technique for the formation of MMMs containing a polymeric matrix, an active pharmaceutical ingredient (API) and molecular sieve (MS X) particles capable of controlling API release and secondly, to highlight electrospinning as a methodology for the fabrication of MMMs of defined size and uniform characteristics relative to previously used casting methods.

Poly(E-caprolactone) (PCL) was chosen as the polymeric matrix due to its previously documented high biocompatibility and Food and Drug Administration (FDA) approval for use in pharmaceutical production. ${ }^{18}$ Ibuprofen was chosen as the model API as it has previously been incorporated into PDMS $^{3}$ and PVDF $^{19}$ casted membranes thereby facilitating comparison between the electrospun MMM's fabricated in this work and those in the literature. Membranes were synthesized with and without a molecular sieve in order to assess the effect of the molecular sieve on API release for the purpose of controlling API release in subsequent membrane iterations. The performance of the membranes with respect to API release kinetics are investigated experimentally and compared to mathematical models commonly used in the field. Fabrication of the electrospun MMMs is discussed in detail and the advantages of electrospun MMMs, based on the integration of theoretical and experimental results are discussed.

\section{Methodology}

\subsection{Membrane preparation}

Mixed matrix membranes were synthesized containing Ibuprofen as an active pharmaceutical ingredient (API) model, poly( $\varepsilon$-caprolactone) (PCL), as the membrane matrix, and the zeolite commonly known as $13 \mathrm{X}$ (molecular sieve $\mathrm{NaX}$ (MS X)) as the inorganic filler. MS X was purchased from Sigma Aldrich (Lot \#MKBR8919V). Membranes were synthesized utilizing two methods, a conventional casting method and through electrospinning. Each method was used to synthesize membranes with and without (MS X), as outlined in Table 3. API was dissolved in $30 / 70$ vol\% ethanol/dichloromethane mixture. For the membranes containing the molecular sieve, dried MS X particles were added to the solution after the API was completely dissolved. After mixing and sonication, PCL was dissolved until the mixture became visually homogeneous in order to generate the precursor of the membranes.

Fig. 1 shows a schematic of the process used for the fabrication of electrospun and casted membranes. For the casting method, approximately $20 \mathrm{ml}$ of the precursor solution was placed in a beaker and allowed to dry at room temperature for 24 hours. It is important to mention that the membranes with MS X, required higher precursor volumes to produce defect-free membranes. Membranes were subsequently stored in an oven at $40{ }^{\circ} \mathrm{C}$ for 24 hours to ensure complete solvent removal followed by hot-pressing at $50{ }^{\circ} \mathrm{C}$ to ensure flatness of the films. Electrospun membranes were generated utilizing a custom built electrospinning device, which consisted of a plastic syringe with a metallic needle. The needle was connected to a high voltage supply (Bertan, Series 230). Grounded aluminium foils were used for collecting the fibres and were located $14 \mathrm{~cm}$ from the needle. Electrospinning parameters were optimised in accordance with solution properties such as polymer molecular weight and solution viscosity. ${ }^{\mathbf{1 1}}$ The precursor solution containing PCL, API and MS X was injected at a flow rate of 50-70 $\mathrm{mL} \mathrm{h}^{-1}$ and deposited utilizing a current of 7-9 $\mathrm{kV}$ onto aluminium foils to form thin fibrous films. A single layer was considered completed when the metallic foil was visually completely covered. Each layer formed over the foil was peeled off and stacked in Petri dishes until the total mass of the stack was of $\sim 0.3 \mathrm{~g}$. The multilayer fibrous films were hot pressed at $50{ }^{\circ} \mathrm{C}$ for $5 \mathrm{~min}$ to form a single membrane. For all membranes synthesized in this work, the presence of the solvent was not observed in any of the characterization methods, including FTIR analysis and drug dissolution.

Table 3 Methods and composition of the membranes synthesized in this work

\begin{tabular}{llll}
\hline Nomenclature & System & $\begin{array}{l}\text { Composition } \\
{[w \mathrm{wt} \%}\end{array}$ & Synthesis method \\
\hline M1 & IBU/PCL & $3 / 97$ & Casting \\
M2 & IBU/MS X/PCL & $3 / 10 / 87$ & Casting \\
M3 & IBU/MS X/PCL & $3 / 20 / 77$ & Casting \\
M1_ES & IBU/PCL & $3 / 97$ & Electrospinning \\
M2_ES & IBU/MS X/PCL & $3 / 10 / 87$ & Electrospinning
\end{tabular}


1) Casting

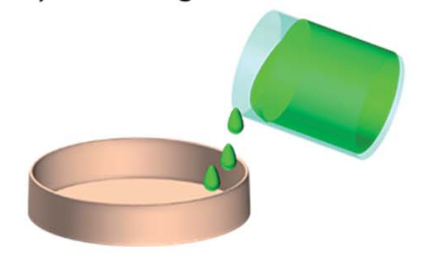

2) Dry and remove

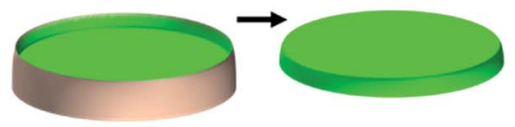

3) Hot Pressing

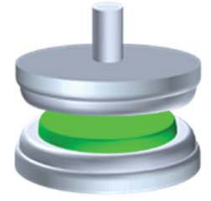

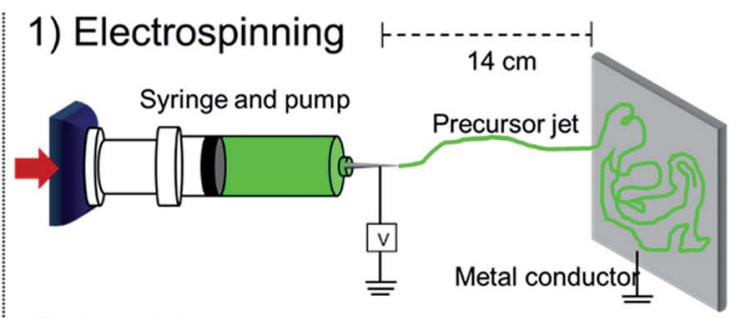

2) Stacking

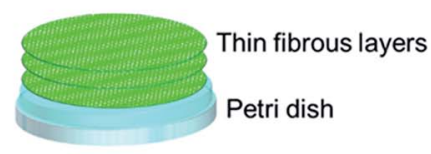

3) Hot Pressing

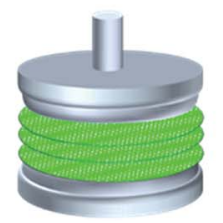

Fig. 1 Schematic of the casting and electrospinning fabrication process.

\subsection{Membrane and materials characterization}

Prior to membrane preparation the particle size distribution of Ibuprofen and the MS X was measured utilizing a Microtrak S3500 particle size analyser; while X-ray powder diffraction (XRD) and Fourier transform infrared spectroscopy (FTIR) were used to determine the main characteristics of the raw materials. To assess membrane dissolution and API release for pharmaceutical applications each film was submerged in a USP conform dissolution basket set-up apparatus type 1 and stirred at $50 \mathrm{rpm}$ for over 24 hours to ensure complete drug dissolution, as specified by the guideline on quality of transdermal patches from the European Medicines Agency. ${ }^{20}$ The dissolution chamber contained $900 \mathrm{~mL}$ of phosphate buffer with a pH 7.2 at $37{ }^{\circ} \mathrm{C}$ for $>24 \mathrm{~h}$. The apparatus used for the test to simulate actual gastric dissolution and the phosphate buffer solution are typically used for the dissolution test of APIs/excipients in the pharmaceutical industry. ${ }^{21}$ The solubility of Ibuprofen in this buffer has been reported to be of $5 \mathrm{~g} \mathrm{~L}^{-1}$ at $20{ }^{\circ} \mathrm{C}$, thereby ensuring that the delivery of the API is not limited by the solubility of the API in the buffer. After taking a sample of $\sim 1.5 \mathrm{ml}$, fresh buffer was injected into the vessel containing the membranes keeping the buffer volume constant through the test. At all instances, it was assumed that the reservoir was well mixed and no concentration gradients were present.

API release was analysed utilizing ultraviolet-visible spectroscopy (UV-Vis) at a wavelength of $220 \mathrm{~nm}$ as MS X is not detectable at this wavelength. Physical characterisation of the membranes was carried out using scanning electron microscopy (SEM) imaging as well as XRD and FTIR. The swelling ratio (SR) and erosion ratios (ER) of the membranes were determined by weighing the membranes before $\left(W_{\mathrm{B}}\right)$, immediately after testing $\left(W_{\mathrm{W}}\right)$ and post-test dried $\left(W_{\mathrm{D}}\right)$; these membrane properties were computed according to eqn (1) and (2).

$$
\begin{aligned}
& \mathrm{SR}=\frac{W_{\mathrm{W}}-W_{\mathrm{B}}}{W_{\mathrm{B}}} . \\
& \mathrm{ER}=\frac{W_{\mathrm{B}}-W_{\mathrm{D}}}{W_{\mathrm{D}}} .
\end{aligned}
$$

\subsection{Mathematical modelling of API release}

A mathematical model was developed in order to evaluate the performance of the membranes generated by both casting and electrospun methods. It is expected that the membranes will follow a conventional diffusion-solution mechanism and thus a deviation of this behaviour could indicate the presence of structural defects and anomalies in the membranes. The developed models will aid in predicting the diffusion coefficient for each of the respective membranes.

Various mathematical models have been proposed in the literature to describe the release profile of soluble API from drug delivery devices, a summary of which has been presented by Costa et al. ${ }^{22}$ Specifically in the case of mixed matrix membranes, Aligieri et al. $^{3}$ applied various models including zero order, first order, Higuchi, Bhaskar and Korsemeyer-Peppas in an attempt to explain the release mechanism of drugs from different mixed matrix membranes. In this work, based on physical considerations, we consider the performance of two diffusion based models to describe the release profile of ibuprofen from both casted and electrospun membranes. These models are the most relevant due to their accuracy in describing the physical phenomena that occurs during drug delivery/API release.

2.3.1. First order model. To begin, we consider a simple model based on first order release kinetics. The average concentration of extractible API in the membrane is denoted by $c(t)$. The membrane volume and cross-sectional area are given by $V$ and $A$ respectively. The extraction of an API is assumed to be driven by the concentration difference between the 
membrane and the buffer solution. Assuming perfect sink conditions the extraction is governed by the equation;

$$
V \frac{\mathrm{d} c}{\mathrm{~d} t}=-2 A k c .
$$

The constant $k$ is the mass transfer coefficient and $c(0)=c_{0}$ gives the initial API concentration. Solving this equation and rewriting it in terms of the fraction of the API extracted we obtain;

$$
\frac{M(t)}{M(\infty)}=1-\mathrm{e}^{-\frac{2 k A}{V} t} .
$$

The quantities $M(t)$ and $M(\infty)$ represent the mass of the API extracted into solution at time $t$ and at full extraction respectively. The mass transfer coefficient can be rewritten in terms of an effective diffusion coefficient $D_{\mathrm{k}}$, and the half thickness of the membrane $H$ as $k=\frac{D_{\mathrm{k}}}{H}$. It is important to note that the effective diffusion coefficient in this equation depends on the average membrane concentration and the choice of diffusion length scale $(H)$. Thus we do not expect it to agree with the diffusion coefficient $D$, detailed in the full diffusion model below which accounts for the concentration variation within the membrane. It is however useful for finding the rate constant for the assumption of first order release, facilitating comparison between membranes and giving an approximate idea of the size of the diffusion coefficient $D$. The data is fitted using the least squares method and assuming that the last experimental data point corresponds to $M(\infty)$ (i.e. all extractable API has been released).

2.3.2. Diffusion model. The second model considers the variation of API concentration along the membrane thickness. It is assumed that the concentration is homogenous in any cross-section of the membrane and extraction from the edges of the membrane is negligible. Thus, the API concentration in the membrane is modelled using the one-dimensional diffusion equation. We let $x$ represent the coordinate along the membrane axis, so the problem domain is $-H<x<H$. We define a symmetry boundary condition at $x=0$ and a perfect sink boundary condition on the membrane surface at $x=H$. Denoting the concentration of extractible API as $c(x, t)$, and the diffusion coefficient as $D$, we have;

$$
\begin{gathered}
\frac{\partial c}{\partial t}=D \frac{\partial c}{\partial x}, \quad 0<x<H, \\
\frac{\partial c(0, t)}{\partial x}=0, \\
c(H, t)=0, \\
c(x, 0)=c_{0} .
\end{gathered}
$$

This problem is readily solvable by separation of variables to yield the API concentration at a given time and axial position in the membrane. ${ }^{23}$ As above, we define $M(t)$ as the mass of API released into the buffer solution at time $t$ and $M(\infty)$ as the mass of extractible API in the membrane. The mass of extracted API at time $t$ is calculated using:

$$
M(t)=H c_{0}-\int_{0}^{H} c(x, t) \mathrm{d} x .
$$

Substituting the solution for $c(x, t)$ into the equation gives the mass fraction of extracted API at time $t$ :

$$
\begin{gathered}
\frac{M(t)}{M(\infty)}=1-\frac{8}{\pi^{2}} \sum_{n=1}^{\infty} \frac{1}{(2 n-1)^{2}} \exp \left(-\frac{(2 n-1)^{2} \pi^{2} D t}{4 H^{2}}\right), \\
t>0 .
\end{gathered}
$$

Thus the equation gives an analytical expression for the mass fraction of API released at time $t$. Experimental data on mass of API extracted is normalised by dividing by the last experimental data point $(M(\infty))$. The equation is then fitted to the data using the least squares method with the diffusion coefficient $D$ being the unknown parameter to be estimated. The models introduced here will be referred to as the first order and diffusion models in the rest of the text.

\section{Results and discussion}

\subsection{Raw material characterization}

Fig. 2 shows the particle size distributions of ibuprofen, used as a model API due to its well-characterised properties, and MS X, where the API displayed a wide range of particle sizes with a mean particle size of $263 \mu \mathrm{m}$. The MS X showed a bimodal particle size distribution with a mean particle size of $463.6 \mu \mathrm{m}$. The surface area of the MS X was reported to be of $832 \mathrm{~m}^{2} \mathrm{~g}^{-1}$. Although, ibuprofen did not display a much smaller particle size than MS X, it is expected that, when mixed with the solvents, its dissolution and subsequent dispersion effectively produced an amorphous solid dispersion (ASD) or a crystal/ASD hybrid. $^{24}$ Consequently, after homogenisation, the solution used to synthesize M2 and M2_ES was dried and XRD of the API/MS-X powders was performed. Fig. 3 shows the XRD spectra

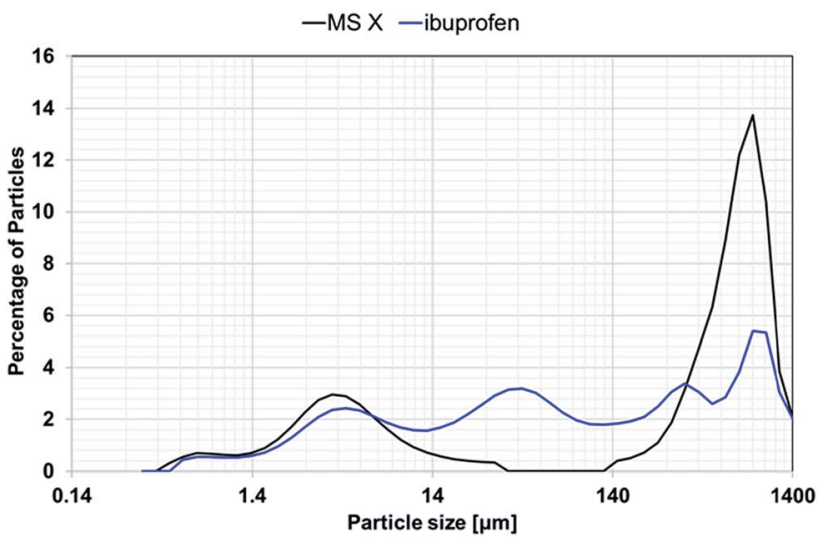

Fig. 2 Particle size distributions for Ibuprofen and the filler MS X, obtained with a Microtrak S3500 particle size analyser. 

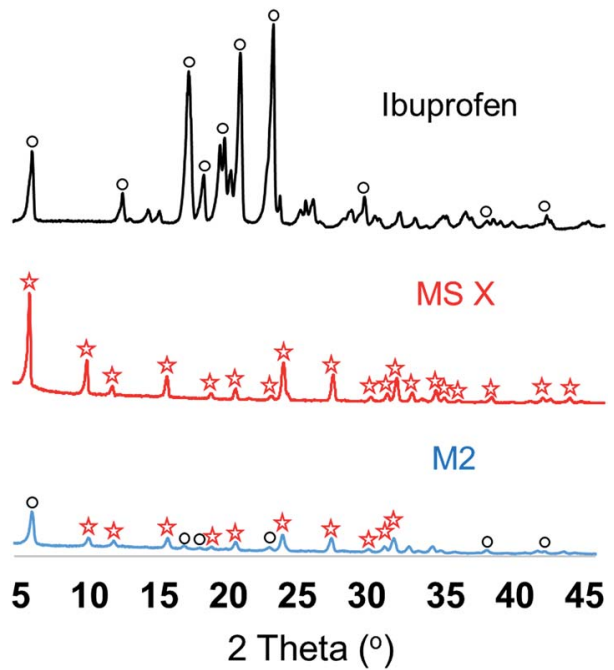

Fig. 3 XRD spectra for ibuprofen, MS X and M2 powder precursor.

of pure Ibuprofen, MS X and the API/MS-X. The characteristic peaks of both components can be identified (MS X and ibuprofen). The intensity of the peaks decreased as ibuprofen may have recrystallized with some lattice variations in its structure and/or have attached to the surface of the molecular sieve. Fig. 4 shows the FTIR analysis of the powders and confirms results shown by the XRD spectra by displaying the characteristic peaks for MS X and ibuprofen. Additionally spectra of the powders used for M2 and M3 clearly indicate the presence of ibuprofen. It is important to mention that M3 shows weaker ibuprofen peaks since the API concentration for these powders is lower than in $\mathrm{M} 2$.

\subsection{Membrane physical properties}

The physical properties of the membrane used in this work are shown in Table 4 . The membranes produced through casting had a reduced diameter than those produced via electrospinning and their properties were diverse. For instance, Fig. 5a shows that membranes M1, M2, and M3 shrunk when the solvent evaporated, yielding, in various occasions, a wrinkly film with uneven thickness. To determine the average thickness of the films, the density and volume of the membranes was measured. At first, the membranes were weighed and their volume estimated by the total amount of liquid displaced when submerged in a solution. After measuring the diameter of the membranes the membrane thickness can be identified. As shown in Table 4, the thickness of the membranes produced through casting varied significantly, since the membranes containing the molecular sieve required more precursor solution to synthesize defect-free membranes. In particular, membranes M3 required almost double the amount of precursor to generate a continuous film. This effect can be rooted to the inorganic-polymer interface, where the detachment of polymeric chains from the inorganic filler causes interfacial voids. There are many hypothesis on what causes interfacial voids including: (i) stresses during solvent evaporation resulting in-filler dewetting or polymer-filler contact; (ii) repulsive forces between
Fig. 4 FTIR for MS X, ibuprofen and powders used for M2 and M3.

Table 4 Membrane characteristics presented in this work

\begin{tabular}{lllll}
\hline Membrane & $\begin{array}{l}\text { Diameter } \\
{[\mathrm{mm}]}\end{array}$ & $\begin{array}{l}\text { Density } \\
{\left[\mathrm{g} \mathrm{ml}^{-1}\right]}\end{array}$ & $\begin{array}{l}\text { Thickness } \\
{[\mathrm{mm}]}\end{array}$ & $\begin{array}{l}\text { Weight } \\
{[\mathrm{g}]}\end{array}$ \\
\hline M1 & 34.1 & 0.45 & 0.67 & 0.19 \\
M2 & 34.1 & 0.39 & 0.68 & 0.19 \\
M3 & 34.1 & 0.47 & 1.31 & 0.45 \\
M1_ES & 43.3 & 0.90 & 0.27 & 0.33 \\
M2_ES & 43.3 & 0.77 & 0.27 & 0.31 \\
\hline
\end{tabular}

polymer and filler; and variance in the thermal expansion coefficients among the materials. ${ }^{25}$ It is important to mention that although membranes M1 and M2 had little deviation, microscopy images revealed defects in their surface. Fig. 5 shows further attributes that varied among the cast-based and electrospun membranes, including the presence of defects and uneven surface features at large and small scales. Among the membranes produced via casting, all of them had a surface with pinholes, but the surface of membrane M1 was smoother and more malleable than those containing the molecular sieve. The mechanical attributes of particulate-polymer composites depend on various factors such as particle size, particle-matrix interface adhesion and particle loading. This was clearly observed in the membranes, where the stiffness of the membranes increased with higher inorganic material loads. Adding inorganic particles to a polymer is a common methodology for reinforcing polymer composites. ${ }^{26}$

The SEM images of membranes M2 and M3 (casting-based synthesis) (Fig. 5b) showed particles of MS X, indicating some particle agglomeration in various sections of the membranes' surface. This could also be the result of sedimentation of the inorganic material. In addition the difference in physical properties between the molecular sieve and the liquid polymer solution may have induced the filler to precipitate during 


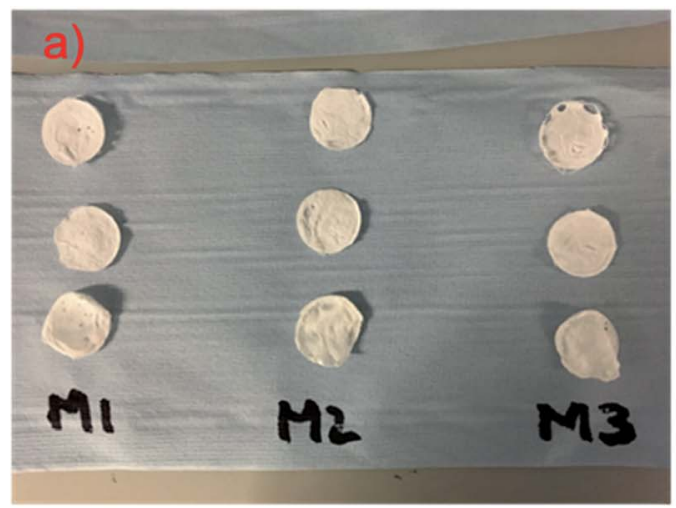

$M 2=$ polymer + MS X $(10 \%)+$ ibuprofen $(3 \%)$

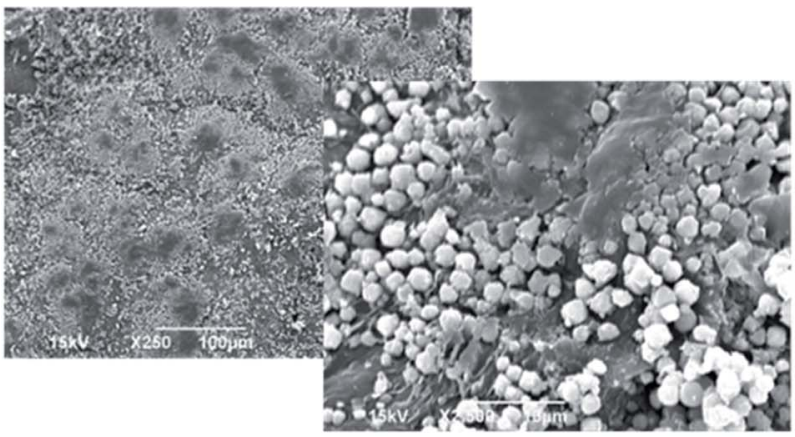

b) $\mathrm{M} 1=$ polymer + ibuprofen $(3 \%)$

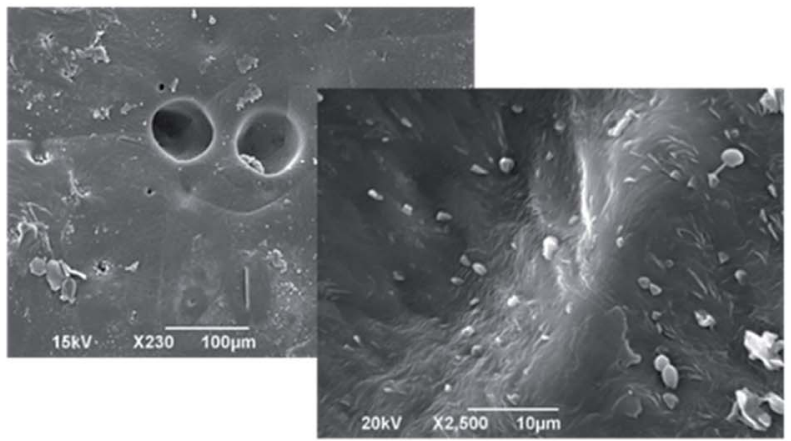

M3= polymer + MS X $(20 \%)+$ ibuprofen $(3 \%)$

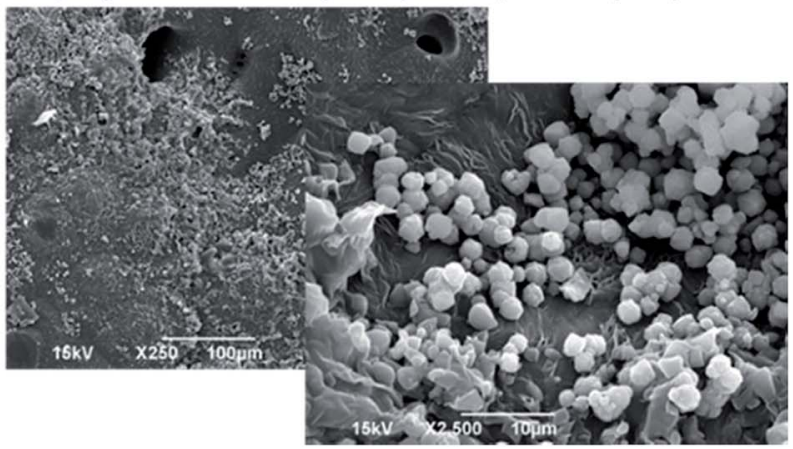

Fig. 5 (a) SEM images and (b) actual membranes produced through the casting method.

membrane formation. It has previously been shown that sedimentation and agglomeration of fillers can cause pinholes in the membrane structure. ${ }^{25}$ Furthermore, the SEM images of M1 suggest the presence of crystals within the structure of the membrane indicating that amorphisation of ibuprofen was not complete and/or unstable within the polymeric matrix. The casting based M1 membrane does not contain a molecular sieve and therefore it can be concluded that some of the ibuprofen may have recrystallized on the membrane surface.

Alternatively electrospun membranes showed a consistent thickness, since the deposition of the fibres was controlled by a layer-by-layer methodology. Typical thickness of the layer electrospun onto the aluminium foil ranged from $5-20 \mu \mathrm{m}$. As each membrane is formed by a layer by layer stacking approach the precise membrane thickness can be easily controlled. As outlined in Table 4 all membranes fabricated via electrospinning had the same thickness and size. Moreover, the membranes fabricated via electrospinning did not show a significant change in their stiffness. A possible reason for this the higher porosity induced by the arrangement of the fibres formed during the electrospinning process as shown in Fig. 6. SEM images in Fig. 6 also reveal that the final hot pressing stage, carried out during the synthesis of the membranes, had no effect on membrane morphology.

SEM images show no visual crystals or signs of API or molecular sieves in crystalline form indicating that ibuprofen and MS X are entrapped within the polymeric fibres with a high degree of amorphicity. The entrapment of ibuprofen into amorphous solid dispersions (ASDs) utilizing PCL was previously demonstrated by Zhu et al., ${ }^{27}$ where microcapsules of PCL/Ibuprofen were generated with a load of $>20 \mathrm{wt} \%$. In this work, X-ray diffraction and FTIR of the membranes was performed to assess crystallinity and/ or amorphous state of ibuprofen within the polymeric membrane. Unfortunately, the detection of the API at a concentration of $3 \%$ was not possible most likely due to low API concentration and entrapment of API within the polymeric fibres. As shown in Fig. 7, some of the peaks of ibuprofen overlap those of the MS X and PCL, and therefore its detection and crystallinity assessment via XRD was also hindered. Nevertheless, it is important to mention that in contrast with the membranes synthesized via casting, electrospun membrane showed no traces of surface MS X and ibuprofen on the SEM images. As a result, it is possible to conclude that the particles were successfully entrapped within the fibres of the membranes however further studies are required to assess the $\%$ crystallinity.

\section{3. $\quad$ API release and mass transfer properties}

Fig. 8 and 9 show the fraction of Ibuprofen released by the membrane at different time intervals. As mentioned previously, the fraction was obtained by normalizing the drug release with the maximum value observed at steady state. All membranes displayed a similar release trend. During the first $30 \mathrm{~min}$, a rapid release is observed that gradually plateaus before reaching a steady maximum value. Results show that electrospun membranes have a faster initial API release rate than membranes produced via casting. For instance, when comparing membranes 
M1= polymer + ibuprofen $(3 \%)$,

a)
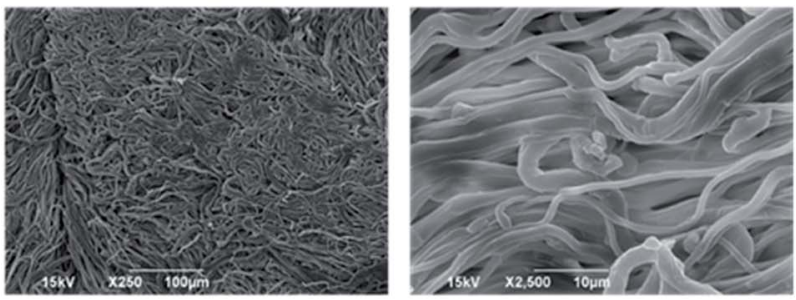

b)
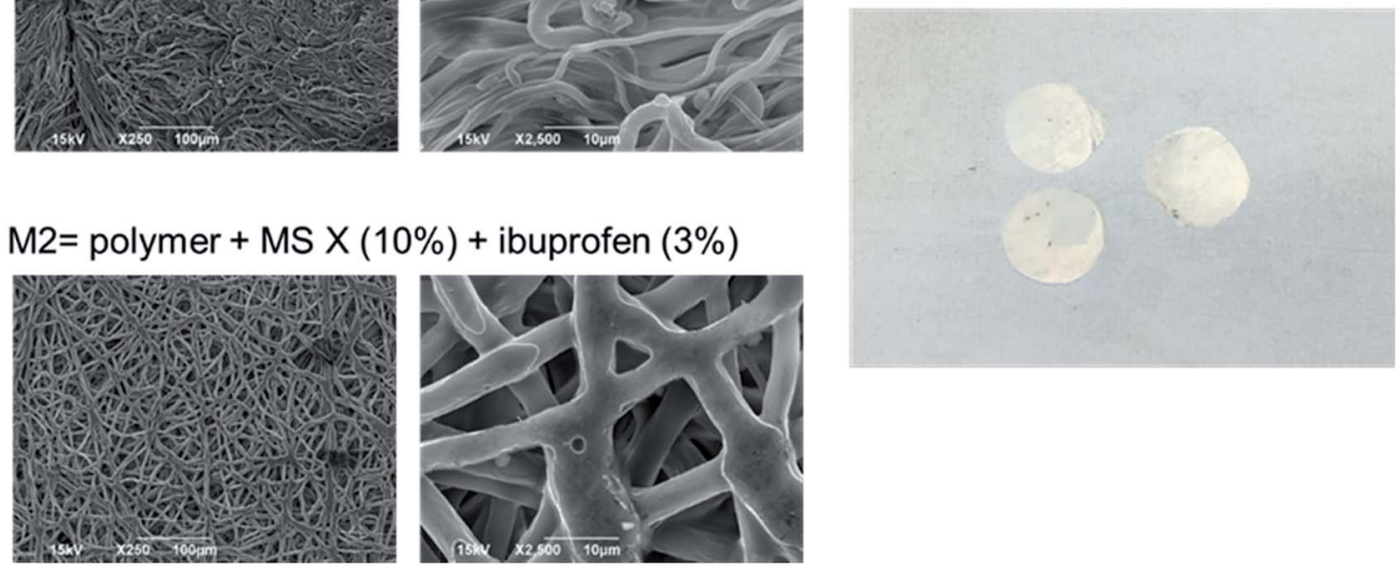

Fig. 6 (a) SEM images and (b) actual membranes produced through electrospinning.

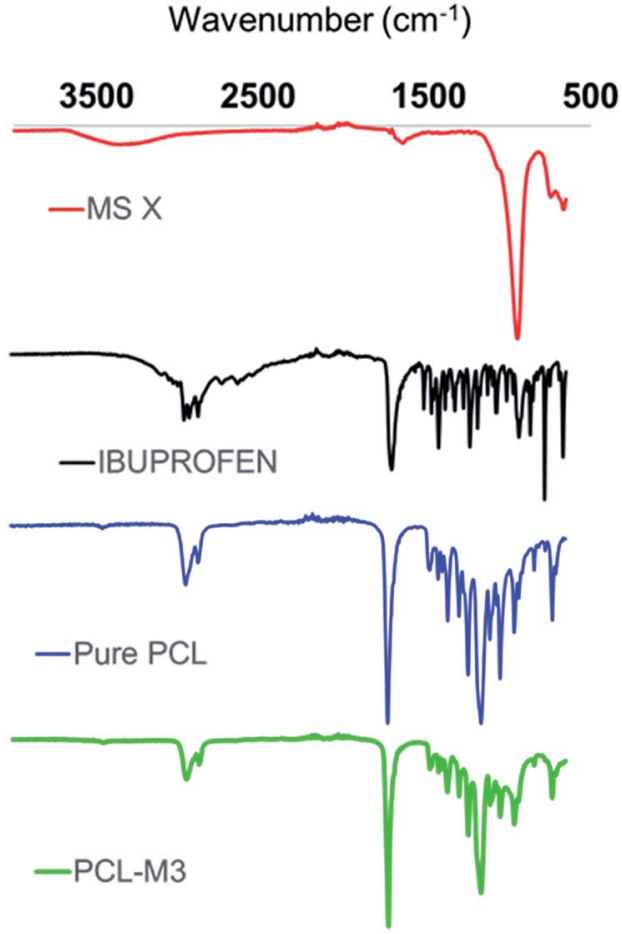

Fig. 7 FTIR spectra of pure precursor components and membrane M3.

M1 and M1_ES, it is possible to notice that after a relatively short time under extraction ( $250 \mathrm{~min}$ ), M1 had released $50 \%$ of the total API, whereas M1_ES had released 75\%. However, at extended times, both membranes reached a maxima of $>90 \%$. The reason for this may be due to the morphology of the electrospun membrane itself. The membrane is thinner and significantly more porous than the membranes formed via casting resulting in significantly increased surface area and improved API release. In addition SEM images of the electrospun membrane revealed no visible API crystallisation suggesting increased API amorphicity within the membrane and hence increased dissolution. It is suspected that some of the ibuprofen remained confined within the polymeric chains of the matrix; up to the point that the thicker membrane M1 appeared to keep releasing small amounts of the API after 24 hours and 100\% extraction was never reached.

It is hypothesized that the transport of the API in electrospun membranes occurs in the following steps: (1) API molecules, encapsulated by a single polymer fibre, diffuse through the interstitial voids of the polymer surrounding it. The API molecule would be required to move a maximum distance equal to the radii of the fibre. (2) At the surface the fibres, the API is desorbed and diffuses into a porous region contained within the membrane "scaffold". (3) The API diffuses outside the membrane scaffold into the buffer solution.

The effect of the molecular sieve on the release of ibuprofen was similar for both casted and electrospun membranes. In both cases the presence of the molecular sieve appeared to hinder the release of the API. For instance, the membrane M3, which contains $20 \%$ of the MS X, showed a maximum extraction of 0.6 (Fig. 8), while membranes M1 and M2 reached 0.9. This effect was not clearly seen when comparing membranes M1 and M2. This could be the due to many variables that could not be tightly controlled in the casting-based membranes such as thickness, porosity and sedimentation/segregation of the API. In the case of electrospun membranes, the physical dimensions were the same and therefore a more conclusive observation can be achieved. Membrane M1_ES reached a maximum extraction of 0.9 , while membrane M2_ES (containing $10 \%$ of the MS X) reached a maximum of 0.7 , suggesting that the MS X has an adsorptive influence to the ibuprofen molecule. The Ibuprofen binds strongly to zeolites with high aluminium content, mainly 

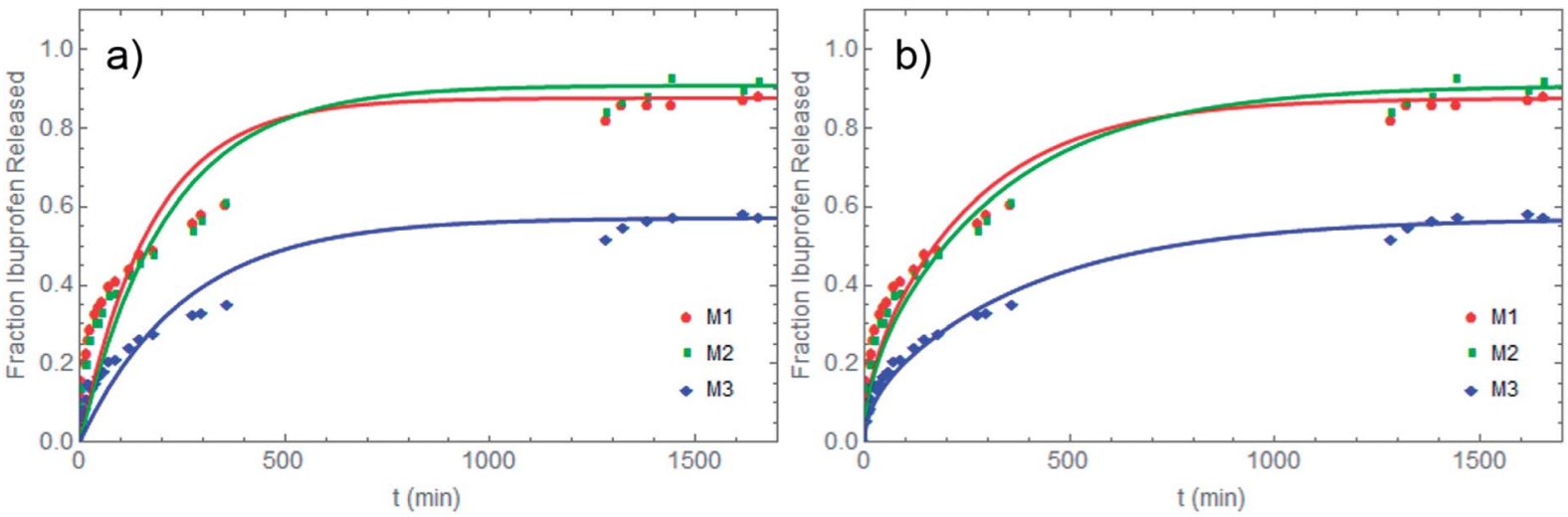

Fig. 8 Ibuprofen release profiles of casted membranes at different times. (a) First order model (solid line) fitted to release data from cast membranes M1, M2 and M3. (b) Diffusion model (solid line) fitted to release data from cast membranes M1, M2 and M3.8.
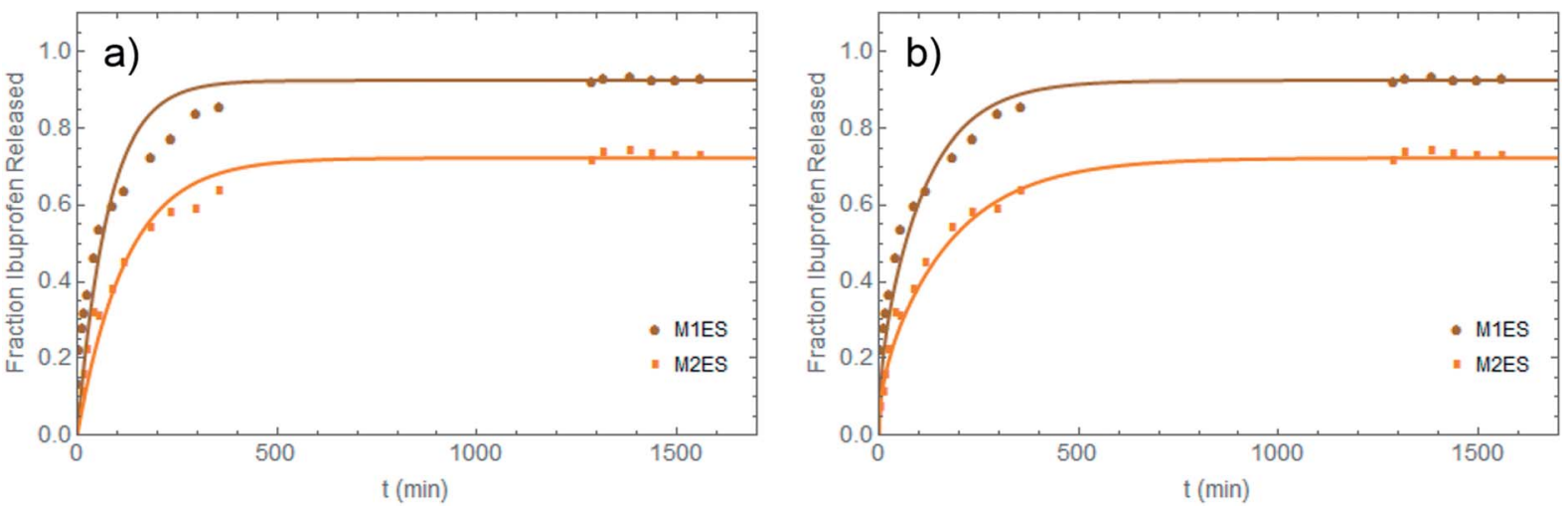

Fig. 9 Ibuprofen release profiles of electrospun membranes at different times. (a) First order model (solid line) fitted to release data from electrospun membranes M1_ES and M2_ES. (b) Diffusion model (solid line) fitted to release data from electrospun membranes M1_ES and M2_ES.

due to an increase in Van der Waals interactions. ${ }^{28}$ Furthermore, ibuprofen, with a kinetic diameter of $6-10 \AA$, is not only entrapped by the polymeric matrix, but also by MS X, which has large pores of $10-13 \AA \AA^{28,29}$ thereby permitting a more controlled/ steady release of the API. The optimal ratio of molecular sieve to API ratio has not yet been reported in the literature for this system.

The experimental release of ibuprofen was fitted with the mathematical models described in Section 2.3 and their performance shown in Fig. 8, 9 and Table 5. At first, it is important to notice that the maximum API extractable from the membrane appeared to be heavily dependent on the zeolite content. Nevertheless, this trend is not consistent between membranes M1 and M2 due to the presence of structural defects in the surface of the membrane. Both, first order and diffusion models showed a reduced $R^{2}$ for these two membranes, although the values still indicated a good overall fit. This may indicate that, in fact, the membranes had structural defects that produced variations in their performance. On the other hand, electrospun membranes showed the highest $R^{2}$ among the membranes tested, demonstrating that their performance follows the predicted mathematical specifications and highlights the more ideal performance of electrospun membranes where API release is concerned. It is apparent that despite their underlying assumptions, the two models applied here can accurately capture the release behaviour of the membranes. The estimated diffusion coefficients are in the order of $10^{-8}-10^{-7} \mathrm{~cm}^{2} \mathrm{~s}^{-1}$ for the cast membranes and $10^{-9}$ $10^{-8} \mathrm{~cm}^{2} \mathrm{~s}^{-1}$ for the electrospun membranes. It is noted that, while the fitted diffusion coefficients are different for the different models as expected, the variation across the membranes in the coefficients follows the same trend.

\subsection{Physical characteristics of membranes after extraction}

The swelling ratio and the extent of membrane erosion were estimated for all membranes utilizing eqn (1) and (2). The weight of the membranes was recorded immediately before and after the dissolution test and after drying for 24 hours in an oven at $40{ }^{\circ} \mathrm{C}$. Fig. 10 shows that the swelling ratio of the membranes produced through the casting method is more than two times higher than those produced via electrospinning. This indicates that although electrospinning produces membranes with intrinsic porosity due to the fibres arrangement, the defects found in the casting-based membranes are higher. This suggests that membrane fabrication via electrospinning 
Table 5 Fraction of extractible API and fitted diffusion coefficients and coefficients of determination for membranes using first order and diffusion models. The initial mass of the API in the membrane is denoted by $M_{0}$

\begin{tabular}{llllll}
\hline & $\frac{M(\infty)}{M_{0}}$ & $\begin{array}{l}D_{\mathrm{k}}\left(\mathrm{cm}^{2} \mathrm{~s}^{-1}\right) \\
\text { (first order) }\end{array}$ & $R^{2}$ & $\begin{array}{l}D\left(\mathrm{~cm}^{2} \mathrm{~s}^{-1}\right) \\
\text { (diffusion) }\end{array}$ & $R^{2}$ \\
\hline M1 & 0.88 & $1.03 \times 10^{-7}$ & 0.967 & $2.71 \times 10^{-8}$ & 0.991 \\
M2 & 0.90 & $9.04 \times 10^{-8}$ & 0.971 & $2.36 \times 10^{-8}$ & 0.994 \\
M3 & 0.57 & $2.83 \times 10^{-7}$ & 0.978 & $7.26 \times 10^{-8}$ & 0.996 \\
M1_ES & 0.92 & $4.00 \times 10^{-8}$ & 0.989 & $1.07 \times 10^{-8}$ & $>0.998$ \\
M2_ES & 0.72 & $2.52 \times 10^{-8}$ & $>0.995$ & $6.98 \times 10^{-9}$ & $>0.998$ \\
\hline
\end{tabular}

provides significantly more control on the design/configuration and the porosity of the membranes. Furthermore, it is clearly shown that higher concentrations of the inorganic filler influenced the swelling ratio. Donato et al. ${ }^{\mathbf{1 6}}$ showed a similar conclusion, where an increase from 12 to $20 \mathrm{wt} \%$ of the $\mathrm{NaX}$ zeolite caused a decrease of the swelling. It is hypothesized that as the inorganic fillers are introduced into the polymer, the polymeric chains reduce their flexibility, causing a net reduction to the interstitial polymeric voids (free volume) and which finally results in a lower sorption ability. The erosion of the membranes was found to be relatively small compared to the swelling ratio. Membranes M1 to M3 presented an erosion lower than 0.03, while electrospun membranes showed a higher erosion (0.05). Even though, erosion was higher for membranes M1_ES and M2_ES, these values are lower than those reported in the literature. ${ }^{16}$ The reduced swelling ratio and erosion of electrospun membranes makes them ideal candidates for their implementation as transdermal patches. Furthermore, the UV spectra of the dissolution tests showed no other components but Ibuprofen, suggesting that all the membranes produced in this worked did not showed impurities and/or degradation.

An additional advantage of fabricating membranes, such as the one proposed in this work, is the formation of amorphous solid dispersions (ASDs). The de-crystallization of Ibuprofen and further dispersion of the molecules within polymers has been reported to improve the release of the drug in the body. ${ }^{30}$

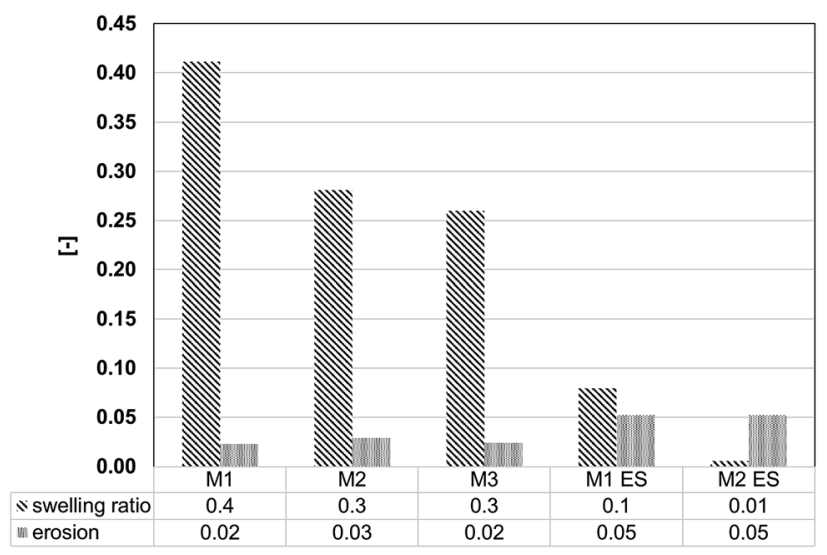

Fig. 10 Physical properties of the membranes during and after testing.
ASDs are used as a strategy to increase the bioavailability of poorly water-soluble APIs such as Ibuprofen, which has a water solubility of $10 \mathrm{mg} \mathrm{L}^{-1}$ at $20^{\circ} \mathrm{C}$. An ASD begins to form when the solid crystalline material is dissolved in a solvent/polymer system and its crystal lattice is disrupted. When the solvent is removed, the API is trapped by the polymer, effectively preventing its recrystallization.

\section{Conclusions}

This work demonstrated the successful development of APIloaded mixed matrix membranes (MMMs) via electrospinning. The fabricated membranes contained: (i) various concentrations of a filler, zeolite 13X; (ii) a model active pharmaceutical ingredient (API), ibuprofen; and (iii) a polymeric matrix, $\operatorname{poly}(\varepsilon$ caprolactone) (PCL). Electrospun membranes were compared with fabrication via casting methodologies widely used in MMM fabrication. The membranes produced in this work exceeded traditional casted based membranes in three performance critical areas: (1) controlled membrane thickness; the thickness of the membranes was based on a layer-by-layer deposition technique permitting the fabrication of MMMs with consistent batch to batch thickness, a significant advantage where the MMM is responsible for delivery of an API. (2) Improved physical properties; electrospun membranes had a reduced swelling ratio relative to those formed via casting. (3) faster and consistent API release features; due to the enhanced control of the membrane fabrication process electrospun membranes showed a faster initial API release rate and demonstrated a better fit with predicted mathematical modelling.

The encapsulation of APIs within inorganic frameworks such as molecular sieves, MOFs and zeolites has been identified as a clear strategy for future drug delivery devices. This work demonstrated that the effect of the inorganic molecular sieve in the membranes synthesized via casting was difficult to evaluate since the properties of the membranes varied significantly. However, within electrospun-based membranes, it is possible to conclude that the molecular sieve slowed the release of the API. The results were supported by two mathematical models, which reflect the ideal performance of the membranes. The successful fitting of the models to the release data indicate a diffusion controlled release from the membranes and diffusion coefficients have been estimated for API release from both cast and electrospun mixed matrix membranes. Although, in vivo dissolution tests are necessary to ensure their commercial applicability, the membranes fabricated via electrospinning displayed more consistent physical characteristics, increased control during the fabrication process and more rapid API release relative to those formed via casting methods. These advantages along with significant potential for scale up highlight the potential of electrospinning as a fabrication technique for MMMs for pharmaceutical and biomedical applications.

\section{Conflicts of interest}

There are no conflicts of interest to declare. 


\section{Acknowledgements}

This work was funded under Science Foundation of Ireland grant "Modelling of Multiphase Transport Automation in Manufacturing, (MOMEnTUM)" - (14/SP/2750). This work was supported by MACSI, the Mathematics Applications Consortium for Science and Industry (www.macsi.ul.ie), funded by the Science Foundation Ireland Investigator Award 12/IA/1683.

\section{References}

1 C. M. Zimmerman, A. Singh and W. J. Koros, J. Membr. Sci., 1997, 137, 145.

2 R. Mahajan and W. J. Koros, Ind. Eng. Chem. Res., 2000, 39, 2692.

3 C. Aligieri, E. Drioli and L. Donato, J. Appl. Polym. Sci., 2013, 5, 754.

4 D. F. Stamatialis, B. J. Papenburg, M. Gironés, S. Saiful, S. N. M. Bettahalli, S. Schmitmeier and M. Wessling, $J$. Membr. Sci., 2008, 308, 1.

5 D. Bastani, N. Esmaeili and M. Asadollahi, J. Ind. Eng. Chem., 2013, 19, 375.

6 E. Pérez, L. Martín, C. Rubio, J. S. Urieta, E. Piera, M. Á. Caballero, C. Téllez and J. Coronas, Ind. Eng. Chem. Res., 2010, 49(18), 8495.

7 T. deV Naylor, Rapra Review Reports, November 1996.

8 R. Gopal, S. Kaur, Z. Ma, C. Chan, S. Ramakrishna and T. Matsuura, J. Membr. Sci., 2006, 281, 581.

9 R. S. Barhate and S. Ramakrishna, J. Membr. Sci., 2007, 296, 1.

10 K. H. Shin, Y. H. Koh and H. E. Kim, Nanomaterials, 2013, 2013, 1 .

11 Z. M. Huang, Y. Z. Zhang, M. Kotaki and S. Ramakrishna, Compos. Sci. Technol., 2003, 63, 2223.

12 C. Shao, H. Y. Kim, J. Gong, B. Ding, D. R. Lee and S. J. Park, Mater. Lett., 2003, 57, 1579.

13 S. Cheng, D. Shen, X. Zhu, X. Tian, D. Zhou and L. J. Fan, Eur. Polym. J., 2009, 45, 2767.

14 R. Ostermann, J. Cravillon, C. Weidmann, M. Wiebcke and B. M. Smarsly, Chem. Commun., 2011, 47, 442.
15 L. Donato, L. Guzzo, E. Drioli and C. Algieri, J. Appl. Polym. Sci., 2015, 132, 41698.

16 L. Donato, G. Barbaro, E. Drioli and C. Algieri, J. Membr. Sep. Technol., 2012, 1, 137.

17 G. Hota, B. R. Kumar, W. J. Ng and S. Ramakrishna, J. Mater. Sci., 2008, 43, 212.

18 B. D. Ulery, L. S. Nair and C. T. Laurencin, J. Polym. Sci., Part B: Polym. Phys., 2011, 49, 832.

19 H. Salazar, A. C. Lima, A. C. Lopes, G. Botelho and S. Lanceros-Mendez, Colloids Surf. A., 2015, 469, 93.

20 European Medicines Agency, Guideline on quality of transdermal patches, http://www.ema.europa.eu/docs/ en_GB/document_library/Scientific_guideline/2014/12/ WC500179071.pdf.

21 U.S. Food \& Drug Administration, Dissolution Methods, https://www.accessdata.fda.gov/scripts/cder/dissolution/ dsp_getallData.cfm.

22 P. Costa and J. M. Sousa Lobo, Eur. J. Pharm. Sci., 2001, 13, 123.

23 J. Crank, The Mathematics of Diffusion. s.l., Oxford Science Publications, 1979, p. 0198534166.

24 P. J. Marsac, T. Lim and L. S. Taylor, Pharm. Res., 2009, 26, 139.

25 T. S. Chung, L. Y. Jiang, Y. Li and S. Kulprathipanja, Prog. Polym. Sci., 2007, 32, 483.

26 S. Y. Fu, X. Q. Feng, B. Lauke and Y. W. Mai, Composites, Part $B, 2008,39,933$.

27 K. J. Zhu, Y. Li, H. L. Jiang, H. Yasuda, A. Ichimaru, K. Yamamoto, P. Lecomte and R. Jerome, J. Microencapsulation, 2005, 22, 25.

28 P. Horcajada, C. Marquez-Alvarez, A. Ramila, J. PerezPariente and M. Vallet-Regi, Solid State Sci., 2006, 8, 1459.

29 Sigma-Aldrich, Molecular Sieves - Technical Information Bulletin, http:/www.sigmaaldrich.com/chemistry/chemicalsynthesis/learning-center/technical-bulletins/al-1430/ molecular-sieves.html.

30 P. J. Marsac, T. Lim and L. S. Taylor, Pharm. Res., 2009, 26, 139. 